\title{
Sarcoidosis of the nervous system
}

\author{
W. B. MATTHEWS \\ From the Derbyshire Royal Infirmary
}

Sarcoidosis of the nervous system can no longer be regarded as a great rarity and has been well reviewed by Jefferson (1957), Garcin (1960), and Suchenwirth (1963), among others. Its protean manifestations are not, however, always recognized and its natural history remains, in many respects, obscure.

\section{INCIDENCE}

The true incidence of sarcoidosis and of its clinical variations is difficult to establish, partly because many patients are segregated in specialized clinics but also because the disease may be symptomless throughout its course (Smellie and Hoyle, 1957). Suchenwirth (1963) stated that of 42,707 patients seen in the medical clinics at Kiel in 12 years, 100 were diagnosed clinically as having sarcoidosis and of these eight had nervous involvement. In the careful study by Mayock, Bertrand, and Morrison (1963) of 145 patients in whom the diagnosis was proved histologically, 23 had symptoms probably indicating involvement of the nervous system. The present series of nine cases was drawn from a population of approximately 500,000 over a period of 10 years. Two cases have been excluded. One patient with proven sarcoidosis had a number of epileptic fits during a period of gross hypercalcaemia without evidence of sarcoid lesions of the nervous system. A futher patient with multiple symmetrical polyneuritis and no systemic evidence of sarcoidosis showed suggestive but inconclusive histological changes on liver biopsy. Important nervous disease occurring in this population would almost certainly have been referred to hospital, although not necessarily diagnosed, but only a very approximate idea of the incidence can be obtained.

\section{CLINICAL FEATURES}

The clinical presentation may be that of predominantly peripheral nervous disease, of progressive diffuse disease of the brain and spinal cord, or of a single intracranial mass. There is much overlap between these groups and they are probably not based on any important pathological distinction, but the classification is of some practical value.

In the present series there were six patients conforming to an admittedly variable but recognizable pattern of maximal incidence on the peripheral nervous system. Of these, three have been previously described (Matthews, 1959), only one being proved by biopsy, and only the salient features will be summarized here. ${ }^{1}$

\section{CASE SUMMARIES}

CASE 1 A 35-year-old woman was seen in 1954. Over the course of four months she had subjective or objective evidence of involvement of all the cranial nerves except the fourth and sixth, combined with asymmetrical weakness of the limbs and scattered areas of hypalgesia on the trunk. Systemic evidence of sarcoidosis was shown by parotitis, hilar node enlargement, and erythema nodosum. The diagnosis was proved by skin biopsy and she was treated with cortisone. Ten years later she shows evidence of faulty innervation of one side of the face, Argyll Robertson pupils, and progressive pulmonary fibrosis.

CASE 2 A woman of 50 was admitted to hospital in 1955 with pain in the chest and fever. She developed bilateral facial palsies, mental confusion, an ataxic gait, and a large area of painful anaesthesia on the abdominal wall. The hilar nodes were enlarged and she had erythema nodosum. Biopsy was not attempted. She was treated with cortisone and recovered completely apart from residual facial contracture. The hilar node enlargement resolved.

CASE 3 A woman of 25 developed in the course of a few weeks bilateral facial palsies, anosmia, optic neuritis, bulbar palsy, an ataxic gait, and scattered areas of analgesia. She had irido-cyclitis and hilar node enlargement. Biopsy was not attempted. She was treated with prednisolone and recovered completely apart from faulty innervation of one side of the face. The hilar nodes resolved.

Three further examples of this form of the disease, proved by biopsy, have been observed.

${ }^{1}$ By kind permission of the Editor of the British Medical Journal. 


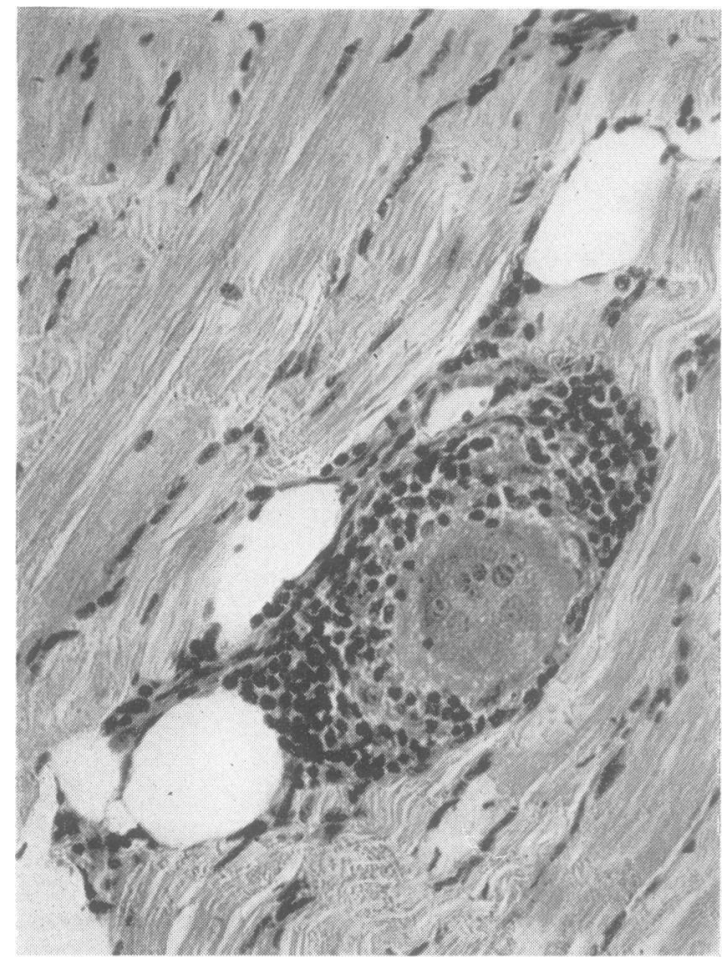

FIG. 1. Biopsy from left quadriceps (case 4) $\times 250$.

CASE 4 (DRI 199341) This 58-year-old woman was seen in October 1958 two days after the onset of a right facial palsy. The palsy was complete and as no other abnormalities could be found it was regarded as an idiopathic Bell's palsy. Nine days later she was admitted to a surgical ward as probably having appendicitis. She was complaining of absolute constipation of several days' duration and of pain over a wide area of the right side of the abdominal wall, but there was no rigidity and she was discharged. Her husband later described a change in personality at this time. She was normally placid but had become subject to violent outbursts of rage.

Fifteen days from the onset of the facial palsy she was again admitted to hospital because of complete inability to swallow. The optic fundi were normal and there was no ocular palsy. There was anosmia on the right. The right side of the face was paralysed and taste was absent on the right side of the tongue. There was complete pharyngeal paralysis and paralysis of the left side of the palate, the left vocal cord, and the left sternomastoid muscle. She was still tender in the right iliac fossa and there was an ill-defined area of analgesia on the abdominal wall. The only abnormality in the limbs was loss of the right ankle jerk. She was morose but not confused.

She had an intermittent fever up to $100^{\circ} \mathrm{F}$. but the E.S.R. was $4 \mathrm{~mm}$. The cerebrospinal fluid contained 50 mg. of protein per $100 \mathrm{ml}$. and 4 lymphocytes per c.mm. The Mantoux reaction was negative to $1: 100$. The chest radiograph showed bilateral hilar lymph node enlarge- ment and reticulation of the lung fields. A biopsy from the left quadriceps muscle showed sarcoid nodules (Fig. 1).

She was fed by gastric tube and treatment with prednisolone was begun four weeks from the first symptom. She improved rapidly and $\mathbf{1 0}$ days later was able to swallow normally and all the cranial nerve palsies had recovered except the facial palsy. She was maintained on prednisolone for six months. At this time the only clinical abnormality was a residual facial weakness. The pulmonary lesions did not, however, resolve and when seen shortly before she left the area three years later there was evidence of progressive pulmonary fibrosis.

CASE 5 This 59-year-old woman was first seen in July 1961 six days after the onset of a left facial palsy. She had felt sick and giddy at the onset but there were no abnormal signs apart from the facial palsy which was complete. She was not seen again until seven weeks later by which time the left facial palsy had completely recovered but a right-sided palsy had developed four weeks after the first. She was admitted to hospital in September 1961 by which time she was complaining of shortness of breath and widespread weakness. She experienced severe continuous pain all over the posterior aspect of the chest. Abnormal neurological signs were, however, confined to a partial right facial palsy with loss of taste on that side and loss of tendon reflexes in the right upper limb. ShE looked wretched and ill but there were no specifio abnormalities on general examination and she had noक् fever.

The E.S.R. was not raised. The Mantoux reaction was negative to $1: 100$. The cerebrospinal fluid contained 96 mg. of protein per $100 \mathrm{ml}$. and 2 lymphocytes per c.mmo The hilar lymph nodes were enlarged but the lung fields were clear. A biopsy from the left deltoid was normal bư an aspiration liver biopsy showed the appearances of sarcoidosis (Fig. 2).

She was treated with prednisolone, reducing eventually to a dose of $10 \mathrm{mg}$. a day. The severe pain improved but did not subside completely and the facial palsy also slowly improved. While on this dose she relapsed in November 1961 and was readmitted to hospital. In addition to the chest pain she had developed pain in the right arm. She was completely constipated, having had no bowel action for 10 days. There was swelling of the right optic disc but no visual disturbance. The tendon reflexes were absent in the right upper limb and in both lower limbs with marked generalized weakness of the former. The plantar reflexes were flexor. The gait was ataxic. There was an area of hypalgesia involving the whole of the posterior chest wall. All forms of cutaneous sensation were reduced distally in the right upper limb with loss of postural sense in the fingers. There was no sensory loss in the lower limbs. The cerebrospinal fluid contained $85 \mathrm{mg}$. of protein per $100 \mathrm{ml}$. and no cells were seen.

The dose of prednisolone was increased to $30 \mathrm{mg}$. a day for a few days and then reduced to $20 \mathrm{mg}$. The swelling of the optic disc rapidly subsided and the reflexes in the legs and her gait had returned to normal within two weeks. The strength of the right arm returned much more slowly and was not fully restored until three months later. Constipation remained troublesome for 


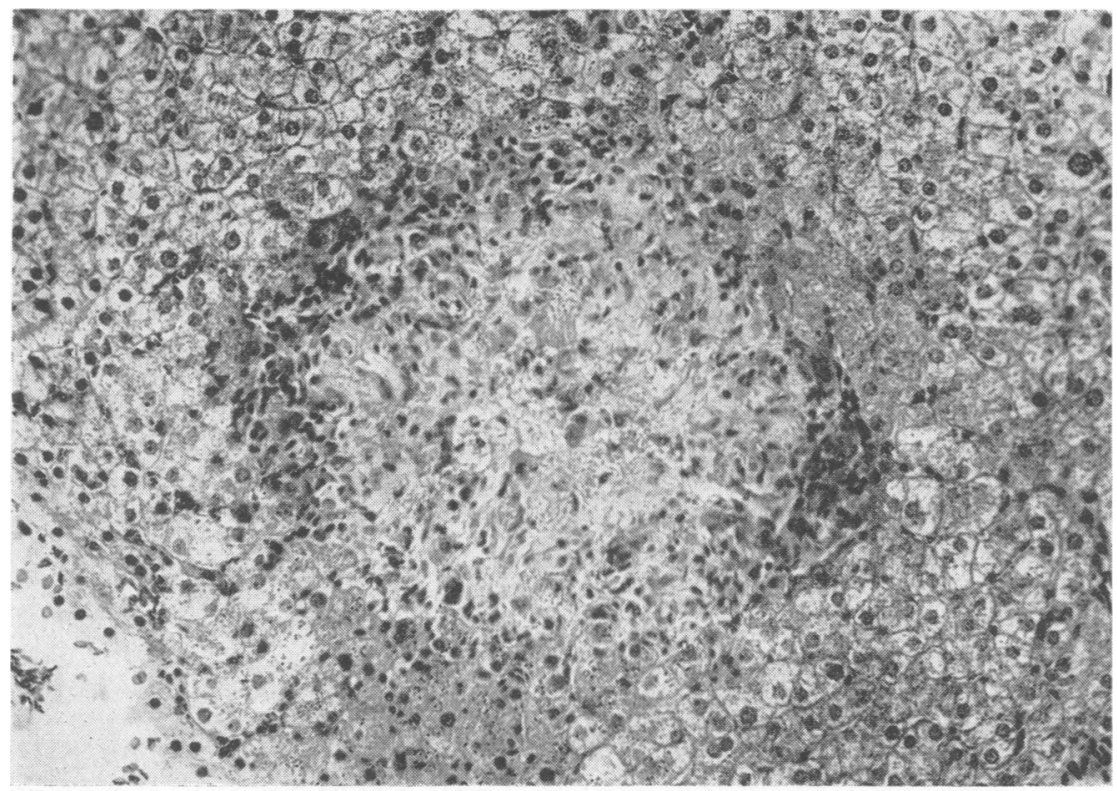

FIG. 2. Aspiration liver biopsy (case 5) $\times 200$.

FIG. 3. Biopsy from gastrocnemius (case 6) $\times 150$.

FIG. 2.

several months and, apart from the residual facial palsy, the most persistent symptom was pain in the chest and around the waist which continued with lessening severity until November 1962. Sensory loss on the trunk of scattered and inconstant distribution could be detected up to this time. Corticosteroid therapy was stopped in December 1962 and she has remained well. The hilar lymph nodes are still enlarged.

CASE 6 (DRI 171540) In January 1961 this 45-year-old woman developed severe pain over a wide area of the dorsal spine and a sensation of numbness round the waist and on the left side of the chest and abdomen. A month later she developed a left facial palsy and was admitted to hospital under the care of Dr. T. R. L. Finnegan. I saw her a few days later and found that she had complete paralysis of the left side of the face with preservation of the sense of taste. There was extensive but ill-defined hypalgesia bilaterally on the trunk. The left ankle jerk was absent but there were no other abnormalities in the limbs. She was aggressive and uncooperative and extremely hyperkinetic. There were no abnormalities on general examination. There was no fever.

The E.S.R. was not raised. The Mantoux reaction

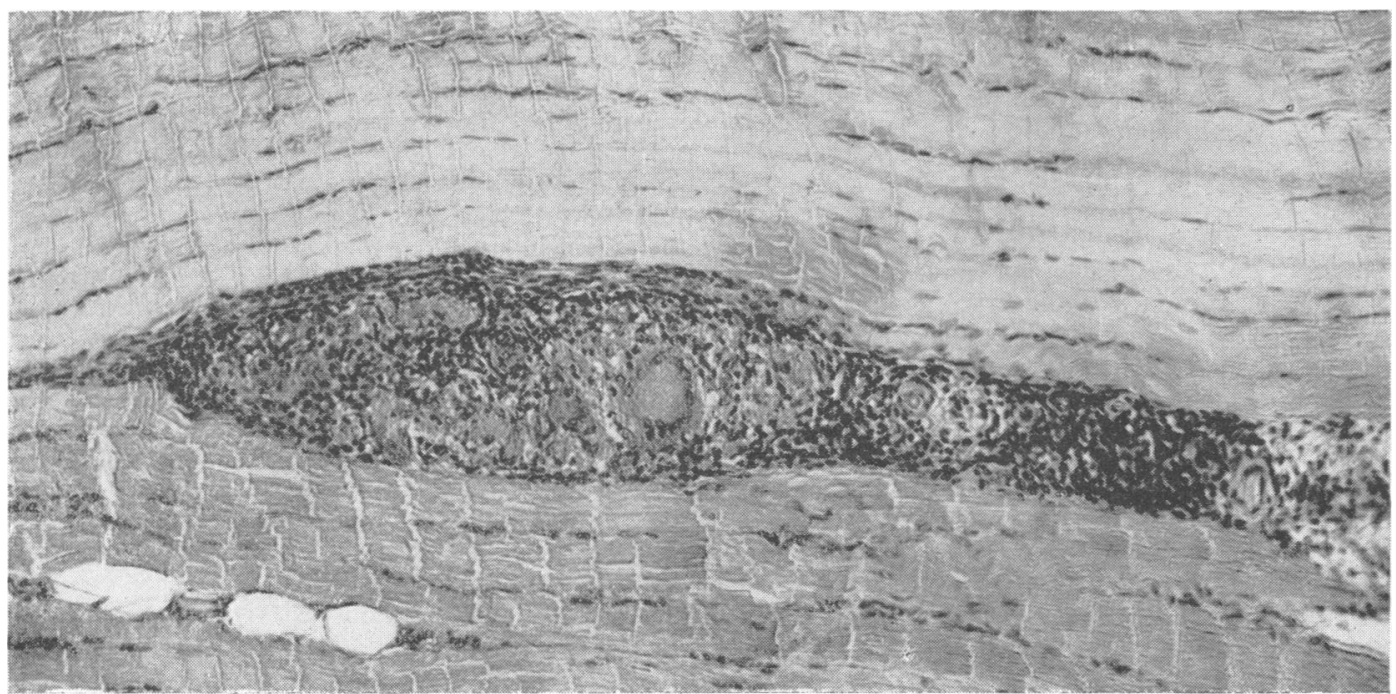

FIG. 3 . 
was negative to $1: 100$. The cerebrospinal fluid contained $90 \mathrm{mg}$. of protein per $100 \mathrm{ml}$. and 18 lymphocytes and 5 polymorphs per c.mm. The hilar lymph nodes were enlarged. A biopsy from the left gastrocnemius showed numerous sarcoid nodules (Fig. 3).

She was treated with prednisolone, $30 \mathrm{mg}$. a day, reducing to a maintenance dose of $15 \mathrm{mg}$. Improvement was steady but not dramatic. Treatment was maintained for six months and when seen in January 1962 the only abnormal finding was a residual facial weakness. She had lost her aggressive attitude and was markedly less hyperkinetic. The pain had resolved. The hilar lymph nodes were reduced in size but still enlarged.

The clinical syndrome in this form of the disease was of an acute or subacute illness with multiple but fluctuating cranial nerve palsies, always including the facial nerve on one or both sides. Evidence of spinal nerve involvement was commonly present but never resembled multiple symmetrical polyneuritis. Sensory symptoms were much more prominent than motor and characteristically included large areas of hypalgesia of bizarre distribution on the trunk, accompanied by pain. This feature has been commented on by Garcin (1960). Neither the character of the pain nor the distribution of the sensory loss resembled those commonly encountered in lesions of the nerve roots. The pain led to a provisional diagnosis of pleurisy in case 2 , of appendicitis in case 5, and of spinal arthritis in case 6 . The distribution, as, for example, the whole of posterior chest wall or one side of the anterior abdominal wall, is more suggestive of involvement of the posterior or anterior primary rami. Prolonged constipation was an important symptom in two patients.

The disease was clearly not confined to the peripheral nervous system. Mental disturbance, confusion, or marked alteration of character was present in three patients and ataxia, apparently of cerebellar type, also in three.

Although these patients often felt very ill, signs of systemic disturbance were slight. Fever was inconspicuous and the E.S.R. was always normal. Clinical evidence of meningitis was not found.

Evidence of sarcoidosis of other organs was present in all patients: iritis in one, parotitis in one, and erythema nodosum in two. A constant feature was bilateral enlargement of the hilar lymph nodes with reticulation of the lung fields in one patient and subsequent pulmonary fibrosis in another. Other well-known signs of sarcoidosis were as consistently absent. No bone lesions were detected; the liver, spleen, and peripheral lymph nodes were not enlarged and there was no hypercalcaemia or disturbance of serum proteins. Hilar node enlargement, uveitis, erythema nodosum, and parotitis are the features encountered in 'primary' pulmonary sarcoidosis, of which hilar node enlargement is the constant factor (Löfgren, 1953). In Europe (Smellie and Hoyle, 1957), but to a lesser degree in America (Sones and Israel, 1960), primary pulmonary sarcoidosis has a good prognosis, the lesions resolving spontaneously in a high proportion of patients, without recurrence. It is known, however, that during the course of this usually benign and sometimes symptomless process sarcoid nodules are certainly present in other organs, notably the liver.

Can the form of sarcoidosis described in these six patients be regarded as a neurological manifestation of the primary form of the disease? If so we would expect a good prognosis in the majority, full recovery and no recurrence, with a small proportion of progressive or recurrent cases. These patients all recovered from their neurological disabilities with the exception of residual evidence of facial palsy and of Argyll Robertson pupils in one patient. Two patients have shown progressive pulmonary fibrosis. All were treated with corticosteroids but full recovery from severe neurological disease of this type, including bulbar palsy, was known before the advent of steroid therapy (Ramsay, 1921). The pathology? of this type of neurological sarcoidosis is obscure as studies have naturally been limited to those with $₫$ fatal outcome. The symptomless dissemination of microscopic sarcoid nodules in liver and muscle i\$ much in favour of similar lesions in the meninges? and possibly the central nervous system rather thang the toxic factor suggested by Mayock, et al. (1963).

If the concept of a primary neurological form of sarcoidosis is correct a small proportion of patients might be expected to develop progressive neurological lesions after the primary phase. The following case history suggests that this may be so.

CASE 7 (DRI 51942) Towards the end of 1960 this 47-year-old woman lost her appetite and began to lose weight. About three months later her eyes became painful and her vision misty. She attended an ophthalmic clinic in March 1961 and was found to have bilateral iritis. This responded only partially to local corticosteroid therapy but it is unlikely that she used the drops regularly. She began to complain of failing memory and was referred to a psychiatric clinic but eventually it became clear that she was suffering from organic nervous disease. When seen in July 1961 she was complaining of loss of balance to the extent of falling, vertigo on lying down, and tinnitus in the right ear.

Her gait had the characteristics of cerebellar ataxia. She was mildly demented as shown by tests of memory and reasoning. There were frequent single myoclonic jerks affecting the right upper limb only. She made no complaint of this but acknowledged the presence of the jerking when her attention was drawn to it. She could not say when it had begun. There were no other abnormal signs. 
There was no past history of epilepsy and the only serious disease in the past had been toxaemia of pregnancy. She had a slight remittent fever up to $100^{\circ} \mathrm{F}$. The cerebrospinal fluid contained $80 \mathrm{mg}$. of protein per $100 \mathrm{ml}$. and 2 lymphocytes per c.mm. The E.S.R. was $4 \mathrm{~mm}$. The Mantoux test was positive to $1: 1,000$. Chest radiographs showed reticulation of the lungs with probable slight hilar node enlargement. The E.E.G. was grossly abnormal. There was a symmetrical alpha rhythm and frequent paroxysms of spike and slow wave discharges at about 4 per second. The myoclonus occurred independently of these paroxyms and was accompanied by single diffuse sharp wave discharges. A biopsy from the left deltoid showed typical sarcoid lesions.

Treatment with prednisolone was begun in August 1961 , reducing to a maintenance dose of $15 \mathrm{mg}$. a day. The iritis rapidly improved and the myoclonus ceased. She regained her balance, began to eat normally and gained weight. The E.E.G. remained abnormal and there appeared to be some residual mental changes as her memory, although improved, was faulty. In March 1962 she stopped treatment against advice and in June she again rapidly lost weight and the iritis relapsed. She developed pain in both sides of the chest and in the right side of the abdomen. She was found to have bilateral optic neuritis with marked blurring of the disc edges. An additional finding was a large area of analgesia involving the abdominal wall on the right side and the anterior aspect of the right thigh. Prednisolone was begun again and she again improved but it is doubtful how regularly she took the treatment. In November 1962 her visual acuity was reduced to $6 / 18$ in both eyes because of lens and vitreous opacities. Treatment again lapsed and when seen again in November 1963 the iritis was active. She was agitated and forgetful and evidently a mild degree of dementia had persisted. The E.E.G. was less abnormal but still contained paroxysms of slow activity without spikes. The reticulation of the lungs remained unchanged and the hilar lymph nodes were thought to be normal.

Here there were features resembling those in the first six patients, notably the painful hypalgesia on the trunk and the dramatic, although partial, response to corticosteroids. There was, however, no facial palsy and cranial nerve lesions were restricted to optic neuritis and tinnitus. The myoclonus and the persistence of mental changes and of the abnormal E.E.G. suggest that central nervous lesions are present and that continuing and perhaps progressive disease is to be feared. The reticulation of the lungs with only slight and resolving hilar node enlargement also suggests that this is a different form or possibly a later stage of the disease.

By no exertions can the majority of reported cases of sarcoidosis of the nervous system be fitted into any neat pattern of primary disease followed immediately or after an interval by progressive lesions. In many, of course, the clinical features described have not been those of the progressive type so that no such history could be expected. In some case reports the history of transient lesions followed by progressive disease is clear enough. For example, Jónasson's (1960) patient had transient diabetes insipidus when aged 23 and cyclitis and enlarged hilar nodes at 25 years of age. Symptoms of progressive paraplegia first appeared at the age of 33 . The patient described by Fine, Gaylor, and Adams (1963), who was seen at the age of 28 with a progressive sarcoid cerebral tumour, gave a history of erythema nodosum and swelling of the knee joints at the age of 11 . The early symptoms of sarcoidosis may be unobtrusive and their significance not appreciated.

CASE 8 (DRI 15654) This woman first attended the neurological clinic in December 1958 at the age of 61 . She was complaining of progressive difficulty in walking since an attack of pneumonia in October 1957. She was found to have asymmetrically increased reflexes in the limbs and extensor plantar reflexes. She had no sensory symptoms or signs. Her speech was noted to be very deliberate. Investigation as an out-patient showed that she had a moderate macrocytic anaemia, histamine-fast achlorhydria, and a megaloblastic bone marrow. Although the clinical features were far from typical a diagnosis of subacute combined degeneration of the spinal cord was quite reasonably entertained. The anaemia responded to treatment with cyanocobalamine but her

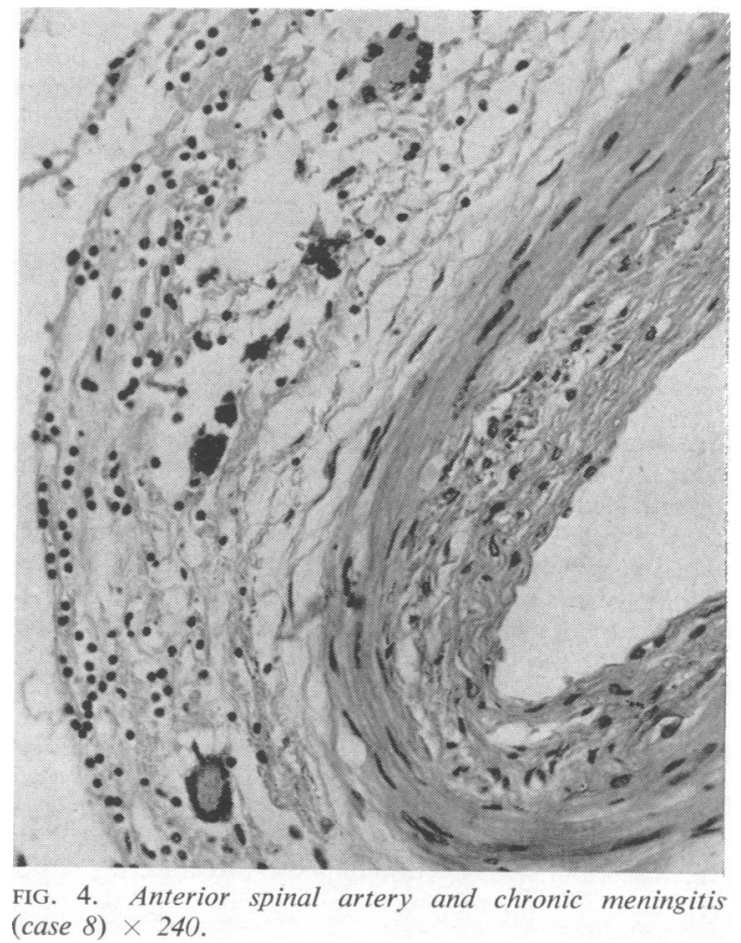


neurological condition did not. Unfortunately the patient was lost sight of as she had been referred by her doctor to a psychiatric clinic because of failing memory and increasing addiction to alcohol.

In February 1960 I saw her again in a hospital for the chronic sick. She was demented and dysarthric and in paraplegia in flexion. No sensory loss could be found. Lumbar puncture showed no evidence of block but the cerebrospinal fluid protein was $200 \mathrm{mg}$. per $100 \mathrm{ml}$. and there were 23 lymphocytes per c.mm. A belated and unsuccessful attempt was made to treat her flexor spasm with intrathecal phenol, the cerebrospinal fluid on this occasion containing $65 \mathrm{mg}$. of protein per $100 \mathrm{ml}$., there being no record of the number of cells. Treatment for pernicious anaemia had continued throughout her illness. She died, undiagnosed, in May 1960 in the chronic sick hospital from infected bed sores.

A necropsy was performed but as she had clearly died from gas gangrene infection of the bed sores no marked attention was paid to the state of the other organs and certainly no evidence of sarcoid was noted. A few routine blocks taken from the central nervous system were, however, available. There was a dense spinal arachnoiditis, not apparently extending over the cerebral hemispheres. Many giant cell systems were present, particularly around the arteries (Fig. 4). No tubercle bacilli were found. The spinal cord did not contain granulations and showed peripheral loss of myelin notably in the lateral columns with relative sparing of the posterior columns.

The appearances were not distinguishable from those of tuberculous meningitis but the clinical history of progressive neurological disease for two and a half years and the absence of tubercle bacilli in an untreated case are much against this diagnosis and suggested the possibility of sarcoidosis. A search for old hospital records revealed that at the age of 48 she had sustained a Bell's palsy followed for a number of years by recurrent iritis.

The extraordinary duration of the evolution of sarcoidosis and the significance of an isolated facial palsy are illustrated in the following case report.

CASE 9 (DRI 61565) This man first attended hospital in June 1951 at the age of 21 with iritis of the right eye. This settled quickly on treatment with local atropine but he attended on several subsequent occasions with milder recurrences. In November 1956 I saw him with a right facial palsy which had developed acutely 10 days earlier. The palsy was complete and accompanied by loss of taste on the anterior portion of the tongue on the affected side. Because of the previous iritis sarcoidosis was suspected but a chest radiograph was normal and the E.S.R. $7 \mathrm{~mm}$. The palsy recovered completely and he was not seen again until February 1963 when he was complaining of cough and headache. On direct questioning he then admitted that since the age of 18 he had suffered from intermittent painful swelling of the joints, particularly the wrists and knees. No swelling was present when he was seen and, indeed, no clinical abnormality could be found. In March 1963 he developed erythema nodosum. A radiograph of the chest showed marked enlargement of the hilar lymph nodes.

He was admitted to hospital but the opportunity of obtaining a biopsy from the skin lesions was missed. Muscle and liver biopsies were normal. He was afebrile. The blood count, E.S.R., serum proteins and calcium, and radiographs of the hands were normal. The cerebrospinal fluid was under normal pressure and was normal in constitution.

He continued to complain of headache and of widespread pains in the trunk and limbs, not localized to the joints. He was treated with prednisolone for seven months at the end of which time he was free from symptoms and the hilar lymph nodes had resolved. No fresh symptoms have appeared.

Here the diagnosis was not confirmed histologically but the occurrence of no less than four manifestations of systemic sarcoid is strongly in favour of the diagnosis, with the facial palsy as the sole evidence of nervous involvement.

\section{DIAGNOSIS}

There is a sharp distinction between the problems of diagnosis in the remittent and the progressive forms of the disease. In the former the occurrence of multiple signs of systemic sarcoid combined with the $-\vec{T}$ characteristic neurological syndrome described above should at once suggest the diagnosis and histologicab proof is not essential. If desired such proof cap usually be obtained from biopsy of muscle or live or of skin lesions when present. The profusion of sarcoid nodules in the skeletal muscle of a patient with no muscular weakness or pain is astonishing.

Little help can be obtained from clinical pathology. The E.S.R. was normal in all patients. The changes in the cerebrospinal fluid were not specific, amounting to no more than a slight pleocytosis and increase in protein. The fluid is sometimes normal at the height of the disease (case 4). The cerebrospinal fluid sugar level has been reported as low in sarcoidosis (Meyer, Foley, and Campagna-Pinto, 1953) but was normal in the four patients in the present series in whom it was examined (cases $1,2,3$, and 6). The serum calcium and proteins were not disturbed. The Mantoux reaction cannot influence the diagnosis as although it is often negative it can be positive in proven sarcoidosis (case 7).

The Kveim test is a method of inducing a positive biopsy and has several disadvantages in the type of case under discussion. The reagent is not universally available, the result is only obtained after considerable delay, and there is some uncertainty as to whether it may be invalidated by corticosteroids.

The diagnosis in the more chronic progressive forms of the disease affecting mainly the centra 
nervous system is exceedingly difficult. It is unlikely that the usual biopsy techniques would always prove successful as even at necropsy no evidence of sarcoidosis outside the nervous system may be found (Reske-Nielsen and Harmsen,' 1962). The diagnosis is, indeed, often unsuspected until operation for symptoms apparently those of a cerebral or spinal tumour. The use of the Kveim test in undiagnosed central nervous disease might prove informative but there is as yet little evidence that it is positive in sarcoidosis clinically or pathologically confined to the nervous system.

\section{TREATMENT}

Corticosteroids were given to eight patients in whom the diagnosis was made in life. It is naturally difficult to assess the results in a disease in which a good prognosis is expected without treatment. Relapse of neurological disease occurred in only two patients. In one the maintenance dose of prednisolone had been reduced to $10 \mathrm{mg}$. a day and the other patient defaulted from treatment. More intensive treatment again resulted in remission. I have no doubt of the efficacy of this treatment in suppressing the disease in its subacute form and that treatment should be continued for some months after complete apparent cure. I have had no opportunity of treating the more chronic forms of nervous sarcoid but there is some evidence that corticosteroids are ineffective (Camp and Frierson, 1962). Why this should be so is obscure, as the fundamental lesion in all forms of sarcoidosis of the nervous system appears to be the microscopic sarcoid nodule.

\section{SUMMARY}

Nine patients with sarcoidosis of the nervous system are described, the diagnosis being demonstrated histologically in six.

Methods of diagnosis, the efficacy of treatment, and the natural history of the disease are discussed.

I am indebted to Dr. T. R. L. Finnegan for permission to publish case 6 .

\section{REFERENCES}

Camp, W. A., and Frierson, J. G. (1962). Sarcoidosis of the central nervous system. Arch. Neurol. (Chic.), 7, 432-441.

Fine, R. D., Gaylor, J. B., and Adams, J. H. (1963). Sarcoid granuloma of brain. Med. J. Aust., 1, 856-860.

Garcin, R. (1960). Les atteintes neurologiques et musculaires dans la maladie de Besnier-Boeck-Schaumann. Psychiat. Neurol. Neurochir. (Amst.), 63, 285-297.

Jefferson, M. (1957). Sarco:dosis of the nervous system. Brain, 80, 540559.

Jónasson, J. V. (1960). Sarcoidosis of the nervous system. Acta psychiat. scand., 35, 182-189.

Löfgren, S. (1953). Primary pulmonary sarcoidosis. Acta med. scand., 145, 424-431.

Matthews, W. B. (1959). Sarcoidosis of the nervous system. Brit. med. J., 1, 267-270.

Mayock, R. L., Bertrand, P., and Morrison, C. E. (1963). Manifestations of sarcoidosis. Amer. J. Med., 35, 67-89.

Meyer, J. S., Foley, J. M., and Campagna-Pinto, D. (1953). Granulomatous angiitis of the meninges in sarcoidosis. Arch. Neurol. Psychiat. (Chic.), 69, 587-600.

Ramsay, A. M. (1921). VI. Diseases of the uveal tract. 1. A case of cyclitis accompanied by swelling of parotid glands and paralysis of cranial nerves. Trans. ophthal. Soc. U.K., 41, 194-209.

Reske-Nielsen, E., and Harmsen, A. (1962). Periangiitis and panangiitis as a manifestation of sarcoidosis of the brain: report of a case. J. nerv. ment. Dis., 135, 399-412.

Smellie, H., and Hoyle, C. (1957). The hilar lymph-nodes in sarcoidosis. Lancet, 2, 66-70.

Sones, M., and Israel, H. L. (1960). Course and prognosis of sarcoidosis. Amer. J. Med., 29, 84-93.

Suchenwirth, R. (1963). Zur Klinik der Meningoencephalitis BesnierBoeck-Schaumann. Arch. Psychiat. Nervenkr., 204, 370-388. 